\title{
Possibilities of the particle finite element method for fluid-soil-structure interaction problems
}

\author{
Eugenio Oñate $\cdot$ Miguel Angel Celigueta • \\ Sergio R. Idelsohn - Fernando Salazar • \\ Benjamín Suárez
}

Received: 28 April 2011 / Accepted: 9 June 2011 / Published online: 8 July 2011

(C) Springer-Verlag 2011

\begin{abstract}
We present some developments in the particle finite element method (PFEM) for analysis of complex coupled problems in mechanics involving fluid-soil-structure interaction (FSSI). The PFEM uses an updated Lagrangian description to model the motion of nodes (particles) in both the fluid and the solid domains (the later including soil/rock and structures). A mesh connects the particles (nodes) defining the discretized domain where the governing equations for each of the constituent materials are solved as in the standard FEM. The stabilization for dealing with an incompressibility continuum is introduced via the finite calculus method. An incremental iterative scheme for the solution of the non linear transient coupled FSSI problem is described. The procedure to model frictional contact conditions and material erosion at fluid-solid and solid-solid interfaces is described. We present several examples of application of the PFEM to solve FSSI problems such as the motion of rocks by water streams, the erosion of a river bed adjacent to a bridge foundation, the stability of breakwaters and constructions sea waves and the study of landslides.
\end{abstract}

Keywords Particle finite element method . Fluid-soil-structure $\cdot$ Interaction

S. R. Idelsohn is a ICREA Professor at CIMNE.

E. Oñate $(\varangle) \cdot$ M. A. Celigueta $\cdot$ S. R. Idelsohn $\cdot$ F. Salazar International Center for Numerical Methods in Engineering (CIMNE), Campus Norte UPC, Gran Capitán s/n, 08034 Barcelona, Spain

e-mail: onate@cimne.upc.edu

URL: http://www.cimne.com

E. Oñate $\cdot$ M. A. Celigueta $\cdot$ B. Suárez

Universitat Politècnica de Catalunya (UPC), Barcelona, Spain

\section{Introduction}

The analysis of problems involving the interaction of fluids, soil/rocks and structures is of relevance in many areas of engineering. Examples are common in the study of landslides and their effect on reservoirs and adjacent structures, off-shore and harbour structures under large waves, constructions hit by floods and tsunamis, soil erosion and stability of rock-fill dams in overspill situations, etc.

These studies can be considered as an extension of the so-called fluid-structure interaction (FSI) problems [46]. Typical difficulties of FSI analysis in free surface flows using the FEM both the Eulerian or ALE formulation include the treatment of the convective terms and the incompressibility constraint in the fluid equations, the modelling and tracking of the free surface in the fluid, the transfer of information between the fluid and the moving solid domains via the contact interfaces, the modeling of wave splashing, the possibility to deal with large motions of multi-bodies within the fluid domain, the efficient updating of the finite element meshes for both the structure and the fluid, etc. Examples of 3-D analysis of FSI problems using ALE and space-time FEM are reported in $[4,6,26,27,31,34,40]$.

Most of the above problems disappear if a Lagrangian description is used to formulate the governing equations of both the solid and the fluid domains. In the Lagrangian formulation the motion of the individual particles are followed and, consequently, nodes in a finite element mesh can be viewed as moving material points (hereforth called "particles"). Hence, the motion of the mesh discretizing the total domain (including both the fluid and solid parts) is followed during the transient solution.

A powerful Lagrangian method for FSI analysis is the so-called Soboran Grid CIP technique, which has been successfully applied to different class of 3-D problems [44]. 
The authors have successfully developed in previous works a particular class of Lagrangian formulation for solving problems involving complex interactions between (free surface fluids) and solids. The method, called the particle finite element method (PFEM, www.cimne.com/pfem), treats the mesh nodes in the fluid and solid domains as particles which can freely move and even separate from the main fluid domain representing, for instance, the effect of water drops. A mesh connects the nodes discretizing the domain where the governing equations are solved using a stabilized FEM.

An advantage of the Lagrangian formulation is that the convective terms disappear from the fluid equations $[11,48]$. The difficulty is however transferred to the problem of adequately (and efficiently) moving the mesh nodes. We use a mesh regeneration procedure blending elements of different shapes using an extended Delaunay tessellation with special shape functions $[17,19]$. The theory and applications of the PFEM are reported in [2,7,10,18,20,21,23,26,32,34-39].

The FEM solution of (incompressible) fluid flow problem implies solving the momentum and incompressibility equations. This is not a simple problem as the incompressibility condition limits the choice of the FE approximations
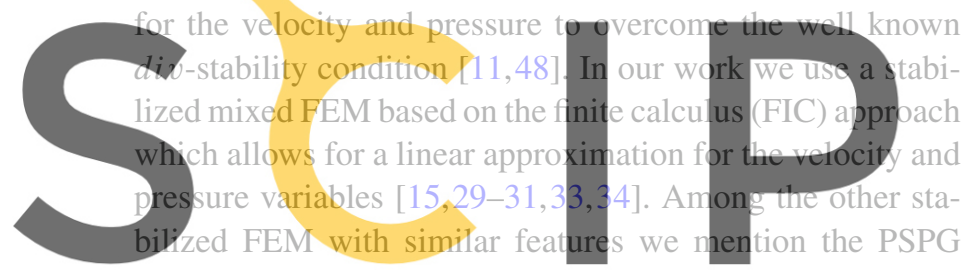

method [41], multiscale methods $[3,6,8,9]$ and the CBS method [9,48].

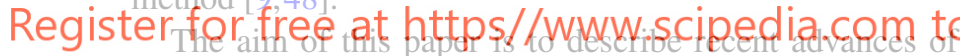

the PFEM for fluid-soil-structure interaction (FSSI) problems. These problems are of relevance in many areas of civil, hydraulic, marine and environmental engineering, among others. It is shown that the PFEM provides a general analysis methodology for treat such complex problems in a simple and efficient manner.

The layout of the paper is the following. In the next section, the key ideas of the PFEM are outlined. Next the basic equations for a compressible/incompressible continuum using a Lagrangian description and the FIC formulation are schematically presented. Then an algorithm for the transient solution is briefly described. The treatment of the coupled FSSI problem and the methods for mesh generation and for identification of the free surface nodes are outlined. The procedure for treating the frictional contact interaction between fluid, soil and structure interfaces is explained. We present several examples of application of the PFEM to solve FSSI problems such as the motion of rocks by water streams, the erosion of a river bed adjacent to a bridge foundation, the stability of breakwaters and constructions under sea waves and the study of landslides falling into reservoirs.

\section{The basis of the particle finite element method}

Let us consider a domain containing both fluid and solid subdomains (the solid subdomain may include soil/rock materials and/or structural elements). The moving fluid particles interact with the solid boundaries thereby inducing the deformation of the solid which in turn affects the flow motion and, therefore, the problem is fully coupled.

In the PFEM, both the fluid and the solid domains are modelled using an updated Lagrangian formulation [47]. That is, all variables are assumed to be known in the current configuration at time $t$. The new set of variables in both domains are sought for in the next or updated configuration at time $t+\Delta t$. The finite element method (FEM) is used to solve the equations of continuum mechanics for each of the subdomains. Hence a mesh discretizing these domains must be generated in order to solve the governing equations for each subdomain in the standard FEM fashion.

The quality of the numerical solution depends on the discretization chosen as in the standard FEM. Adaptive mesh refinement techniques can be used to improve the solution in zones where large motions of the fluid or the structure occur:

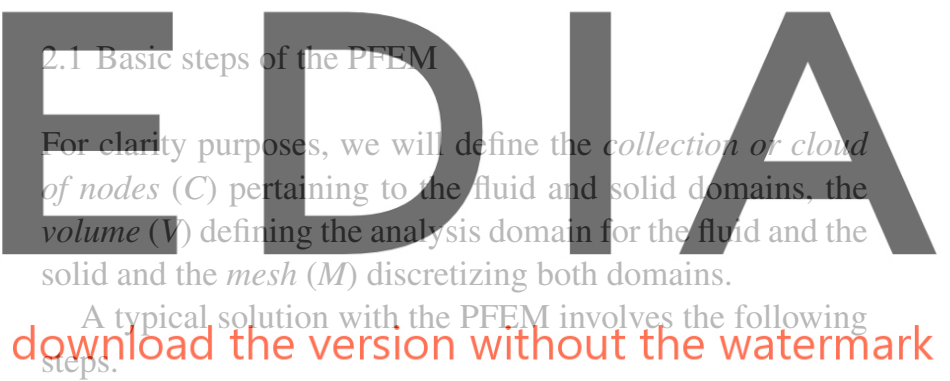

1. The stanting point at each time step is the cloud of points in the fluid and solid domains. For instance ${ }^{n} C$ denotes the cloud at time $t=t_{n}$ (Fig. 1).

2. Identify the boundaries for both the fluid and solid domains defining the analysis domain ${ }^{n} V$ in the fluid and the solid. This is an essential step as some boundaries (such as the free surface in fluids) may be severely distorted during the solution, including separation and re-entering of nodes. The Alpha Shape method [12] is used for the boundary definition.

3. Discretize the fluid and solid domains with a finite element mesh ${ }^{n} M$. In our work we use an innovative mesh generation scheme based on the extended Delaunay tessellation $[17,19,20]$.

4. Solve the coupled Lagrangian equations of motion for the fluid and the solid domains. Compute the state variables in both domains at the next (updated) configuration for $t+\Delta t$ : velocities, pressure and viscous stresses in the fluid and displacements, stresses and strains in the solid. 
Fig. 1 Sequence of steps to update a "cloud" of nodes representing a domain containing a fluid and a solid part from time $n\left(t=t_{n}\right)$ to time $n+2\left(t=t_{n}+2 \Delta t\right)$
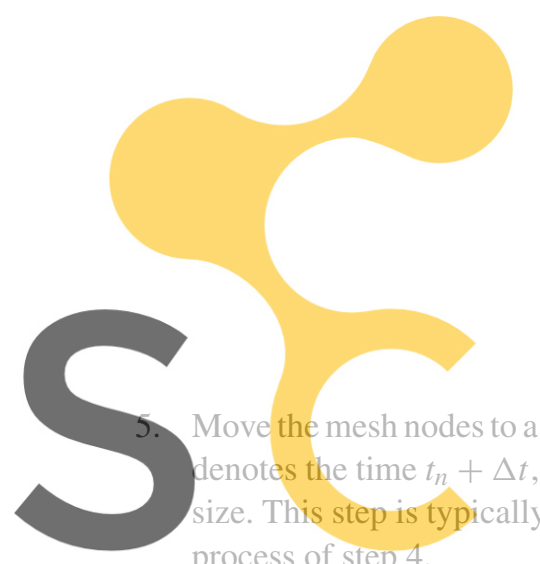
process of step 4

6. Go back to step 1 and repeat the solution process for the Register for fineestatthitpos//Vurviviscipedia.com to

\section{FIC/FEM formulation for a Lagrangian continuum}

\subsection{Governing equations}

The equations to be solved are the standard ones in continuum mechanics, written in the Lagrangian frame of reference:

$$
\begin{aligned}
& \text { Momentum } \\
& \rho \frac{\partial v_{i}}{\partial t}=\frac{\partial \sigma_{i j}}{\partial x_{j}}+b_{i} \quad \text { in } V
\end{aligned}
$$

\section{Pressure-velocity relationship}

$$
\frac{1}{K} \frac{\partial p}{\partial t}-\frac{\partial v_{i}}{\partial x_{i}}=0 \quad \text { in } V
$$

In above equations $v_{i}$ is the velocity along the $i$ th global (cartesian) axis, $p$ is the pressure (assumed to be positive in
- Solid node

O Fluid node

Fixed boundary node
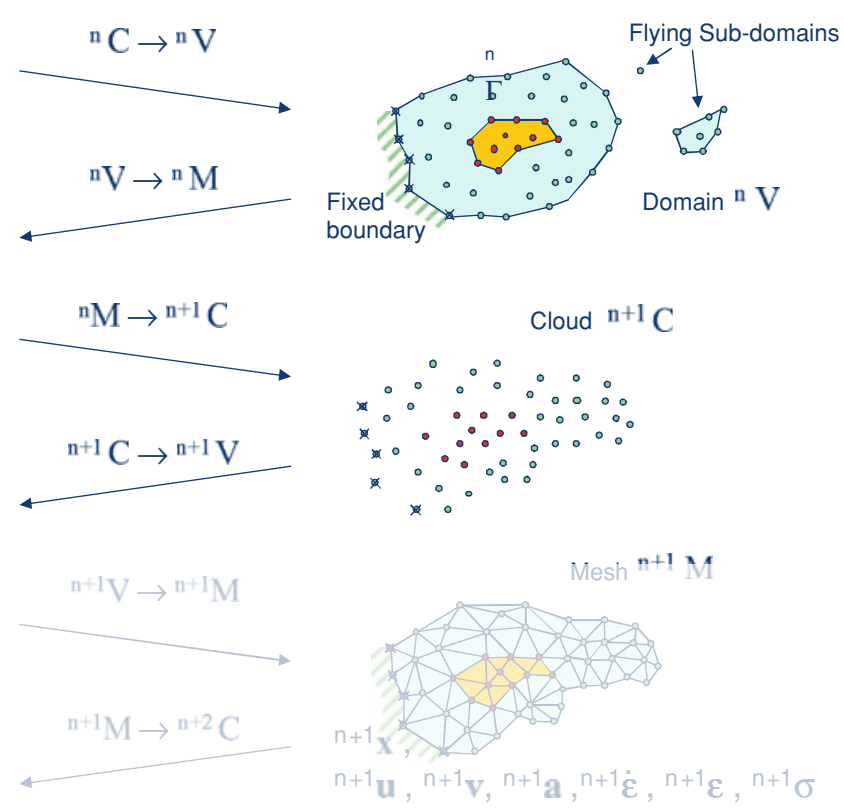

etc...

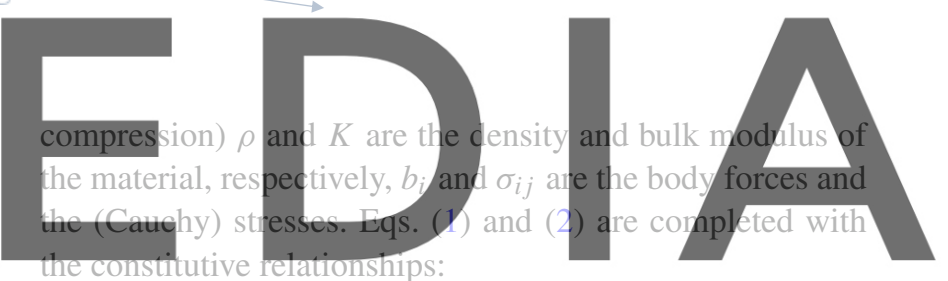

dowmloadbthenviension without the watermark

${ }^{t+1} \sigma_{i j}=2 \mu \dot{\varepsilon}_{i j}-{ }^{t+1} p \delta_{i j}$

Compressible/quasi-incompressible continuum

${ }^{t+1} s_{i j}={ }^{t} \hat{\sigma}_{i j}+2 \mu \dot{\varepsilon}_{i j}+\lambda \dot{\varepsilon}_{i i} \delta_{i j}$

where $\hat{\sigma}_{i j}$ are the component of the stress tensor $[\hat{\sigma}]$

$[\hat{\sigma}]=\frac{1}{J} \mathbf{F}^{T} \mathbf{S F}$

where $\mathbf{S}$ is the second Piola-Kirchhoff stress tensor, $\mathbf{F}$ is the deformation gradient tensor and $J=\operatorname{det} \mathbf{F}[22,47]$. Parameters $\mu$ and $\lambda$ take the following values for a fluid or solid material:

Fluid: $\mu$ : viscosity; $\lambda=\Delta t K-\frac{2 \mu}{3}$

Solid: $\mu=\frac{\Delta t G}{J} ; \lambda=\frac{2 G \nu \Delta t}{J(1-2 v)}$, where $v$ is the Poisson ration, $G$ is the shear modulus and $\Delta t$ the time increment. 
In Eqs. (3) and (4a), (4b), $s_{i j}$ are the deviatoric stresses, $\dot{\varepsilon}_{i j}$ is the rate of deformation, $\mu$ is the viscosity and $\delta_{i j}$ is the Kronecker delta. ${ }^{t}(\cdot)$ denotes values at time $t$.

Indexes in Eqs. (1)-(4a), (4b) range from $i, j=1, n_{d}$, where $n_{d}$ is the number of space dimensions of the problem (i.e. $n_{d}=2$ for 2-D problems). These equations are completed with the standard boundary conditions of prescribed velocities and surface tractions in the mechanical problem $[11,36,47,48]$.

\subsection{Discretization of the equations}

A key problem in the numerical solution of Eqs. (1)-(4a), (4b) is the satisfaction of the mass balance condition for the incompressible case (i.e. $K=\infty$ in Eq. (2)). A number of procedures to solve his problem exist in the finite element literature $[11,48]$. In our approach we use a stabilized formulation based in the so-called finite calculus procedure $[15,29-31,33,34]$. The essence of this method is the solution of a modified mass balance equation which is written as $\frac{1}{K} \frac{\partial p}{\partial t}-\frac{\partial v_{i}}{\partial x_{i}}-\sum_{i=1}^{3} \tau \frac{\partial q}{\partial x_{i}}\left[\frac{\partial p}{\partial x_{i}}+\pi_{i}\right]=0$

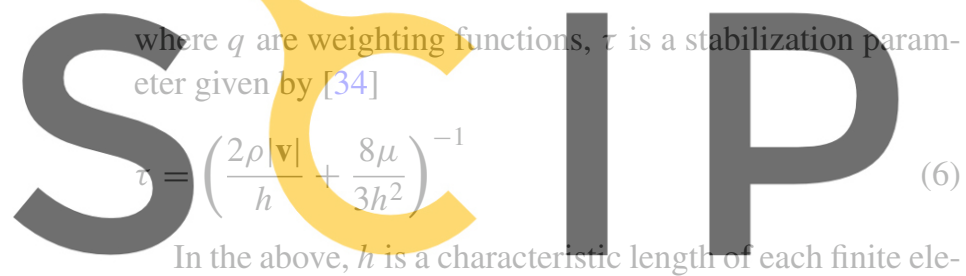
ment and $|\mathrm{v}|$ is the modulus of the velocity vector. In Eq. (5)

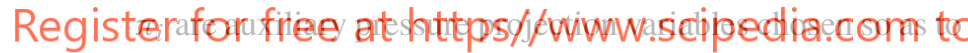
ensure that the second term in Eq. (5) can be interpreted as weighted sum of the residuals of the momentum equations and therefore it vanishes for the exact solution. The set of governing equations is completed by adding the following constraint equation $[32,36]$

$$
\left.\int_{V} \tau w_{i}\left(\frac{\partial p}{\partial x_{i}}+\pi_{i}\right) d V=0 \quad i=1, n_{d} \quad \text { (no sum in }\right) i
$$

where $w_{i}$ are arbitrary weighting functions.

The rest of the integral equations are obtained by applying the standard weighted residual technique to the governing Eqs. (1)-(3) and (5) and the corresponding boundary conditions $[11,22,48]$.

We interpolate next in the standard finite element fashion the set of problem variables. For 3-D problems these are the three velocities $v_{i}$, the pressure $p$, the temperature $T$ and the three pressure gradient projections $\pi_{i}$. In our work we use equal order linear interpolation for all variables over meshes of 3-noded triangles (in 2-D) and 4-noded tetrahedra (in 3-D). The resulting set of discretized equations using the standard Galerkin technique has the following form
Box 1 Basic PFEM algorithm for a Lagrangian continuum

1. LOOP OVER TIME STEPS, $t=1$, NTIME Known values

${ }^{t} \overline{\mathbf{x}},{ }^{t} \overline{\mathbf{v}},{ }^{t} \overline{\mathbf{p}},{ }^{t} \overline{\boldsymbol{\pi}},{ }^{t} \bar{T},{ }^{t} \mu,{ }^{t} \mathbf{f},{ }^{t} \mathbf{q},{ }^{t} C,{ }^{t} V,{ }^{t} M$

2. LOOP OVER NUMBER OF ITERATIONS, $i=1$, NITER

- Compute nodal velocities by solving Eq. (8)

$\left[\frac{1}{\Delta t} \mathbf{M}+\mathbf{K}\right]{ }^{t+1} \overline{\mathbf{v}}^{i+1}={ }^{t+1} \mathbf{f}+G^{t+1} \overline{\mathbf{p}}^{i}+\frac{1}{\Delta t} \mathbf{M}^{t} \overline{\mathbf{v}}$
- Compute nodal pressures from Eq. (9)

$\left[\frac{1}{\Delta t}-\mathbf{L} \overline{\mathbf{M}}\right]^{t+1} \bar{p}^{i+1}=\mathbf{G}^{T t+1} \bar{v}^{i+1}+\mathbf{Q}^{t+1} \overline{\boldsymbol{\pi}}^{i}+\frac{1}{\Delta t} \overline{\mathbf{M}}^{t} \overline{\mathbf{p}}$

- Compute nodal pressure gradient projections from Eq. (10)

${ }^{n+1} \overline{\boldsymbol{\pi}}^{i+1}=-\hat{\mathbf{M}}_{D}^{-1}\left[\mathbf{Q}^{T}\right]^{t+1} \overline{\mathbf{p}}^{i+1}, \hat{\mathbf{M}}_{D}=\operatorname{diag}\left[\hat{\mathbf{M}}_{D}\right]$

- Update position of analysis domain nodes:

${ }^{t+\Delta t} \overline{\mathbf{x}}^{i+1}={ }^{t} \mathbf{x}^{i}+{ }^{t+\Delta t} \mathbf{v}^{i+1} \Delta t$

Define new "cloud" of nodes ${ }^{t+1} C^{i+1}$

- Update strain rate and strain values

- Update stress values

Check convergence $\rightarrow \mathrm{NO} \rightarrow$ Next iteration $i \rightarrow i+1$

$\downarrow$ YES

Next time step $t \rightarrow t+$

- Identify new analysis domain boundary: ${ }^{t+1} \mathrm{~V}$

- Generate mesh: ${ }^{t+1} M$

Go to 1

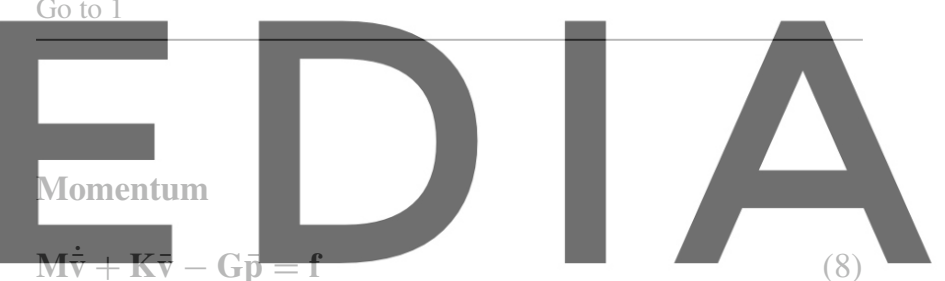

dowaleadithe kensionipwithout the watermark

$\overline{\mathrm{M}} \dot{\overline{\mathrm{p}}}-\mathrm{G} \overline{\mathrm{v}}-\mathrm{L} \overline{\mathrm{p}}-\mathrm{Q} \bar{\pi}=0$

\section{Pressure gradient projection}

$\hat{\mathbf{M}} \overline{\boldsymbol{\pi}}+\mathbf{Q}^{T} \overline{\mathbf{p}}=\mathbf{0}$

In Eqs. (8)-(10) $(\bar{\cdot})$ denotes nodal variables, $\dot{(\bar{\cdot})}=\frac{\partial}{\partial t}(\bar{\cdot})$. The different matrices and vectors are given in $[22,34,36]$.

The solution in time of Eqs. (8)-(10) can be performed using any time integration scheme typical of the updated Lagrangian FEM [36,47]. A basic algorithm following the conceptual process described in Sect. 2 is presented in Box 1.

\section{Generation of a new mesh}

One of the key points for the success of the PFEM is the fast regeneration of a mesh at every time step on the basis of the position of the nodes in the space domain. Indeed, any fast meshing algorithm can be used for this purpose. In our work the mesh is generated at each time step using the so-called extended Delaunay tessellation (EDT) presented in $[17,19]$. 
The CPU time required for meshing grows linearly with the number of nodes. The CPU time for solving the equations exceeds that required for meshing as the number of nodes increases. This situation has been found in all the problems solved with the PFEM. As a general rule for large 3D problems meshing consumes around $15 \%$ of the total CPU time for each time step, while the solution of the equations (with typically 3 iterations to reach convergence within a time step) and the assembling of the system consume approximately 70 and $15 \%$ of the CPU time for each time step, respectively. These figures refer to solutions obtained in a standard single processor Pentium IV PC for all the computations and prove that the generation of the mesh has an acceptable cost in the PFEM. The cost of remeshing is similar to that reported in [24]. Indeed considerable speed can be gained using parallel computation techniques.

\section{Identification of boundary surfaces}

One of the main tasks in the PFEM is the correct definition of the boundary domain. Boundary nodes are sometimes explic-

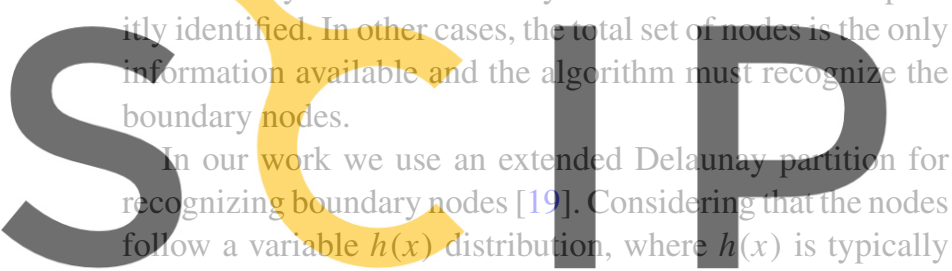

the minimum distance between two nodes. All nodes on an Regisempty sphere with a radius greater than $\alpha$, are considered

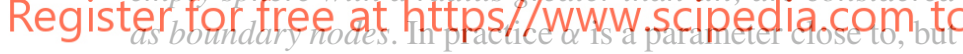
$>$ one. Values of $\alpha$ ranging between 1.3 and 1.5 have been found to be optimal in all examples analyzed. This criterion is coincident with the Alpha Shape concept [12].

Once a decision has been made concerning which nodes are on the boundaries, the boundary surface is defined by all the polyhedral surfaces (or polygons in 2-D) having all their nodes on the boundary and belonging to just one polyhedron.

The method described also allows one to identify isolated fluid particles outside the main fluid domain. These particles are treated as part of the external boundary where the pressure is fixed to the atmospheric value. We recall that each particle is a material point characterized by the density of the solid or fluid domain to which it belongs. The mass which is lost when a boundary element is eliminated due to departure of a node from the main analysis domain is again regained when the "flying" node falls down and a new boundary element is created by the Alpha Shape algorithm.

The boundary recognition method is also useful for detecting contact conditions between the fluid domain and a fixed boundary, as well as between different solids interacting with each other as detailed in the next section.
We emphasize that the main difference between the PFEM and the classical FEM is just the remeshing technique and the identification of the domain boundary at each time step.

\section{Treatment of contact conditions in the PFEM}

\subsection{Contact between the fluid and a fixed boundary}

The condition of prescribed velocities at the fixed boundaries in the PFEM is applied in strong form to the boundary nodes. These nodes might belong to fixed external boundaries or to moving boundaries linked to the interacting solids. Contact between the fluid particles and the fixed boundaries is accounted for by the incompressibility condition which naturally prevents the fluid nodes to penetrate into the solid boundaries $[32,36]$.

\subsection{Contact between solid-solid interfaces}

The contact between two solid interfaces is simply treated by introducing a layer of contact elements between the two inter-

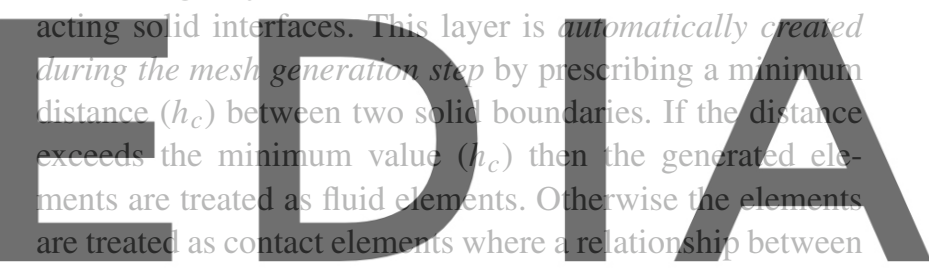

the tangential and normal forces and the corresponding displacement is introduced (Fig. 2i) hout the watermark

to identifying and modeling complex frictional contact conditions between two or more interacting bodies moving in water in an extremely simple manner.

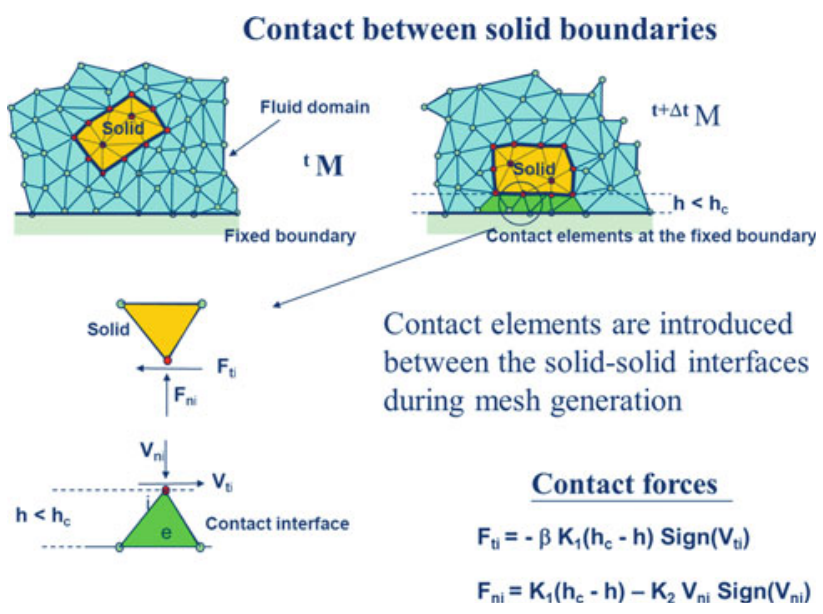

Fig. 2 Modelling of contact conditions at a solid-solid interface with the PFEM 
This algorithm can also be used effectively to model frictional contact conditions between rigid or elastic solids in structural mechanics applications $[7,36]$.

\section{Modeling of bed erosion}

Prediction of bed erosion and sediment transport in open channel flows are important tasks in many areas of river and environmental engineering. Bed erosion can lead to instabilities of the river basin slopes. It can also undermine the foundation of bridge piles thereby favouring structural failure. Modeling of bed erosion is also relevant for predicting the evolution of surface material dragged in earth dams in overspill situations. Bed erosion is one of the main causes of environmental damage in floods.

Bed erosion models are traditionally based on a relationship between the rate of erosion and the shear stress level [25]. In a recent work we have proposed an extension of the PFEM to model bed erosion $[35,36]$. The erosion model is based on the frictional work at the bed surface originated by the shear stresses in the fluid. The resulting erosion model resembles Archard law typically used for modeling abrasive wear in

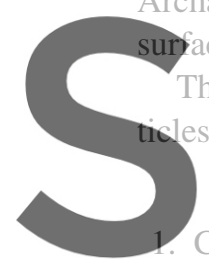

The algorithm for modeling the erosion of soil/rock par-
es at the fluid bed is the following:
Compute at every point of the bed surface the resultant tangential stress $\tau$ induced by the fluid motion. In 3-D

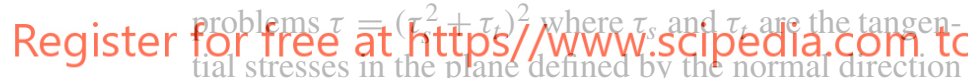
$\mathrm{n}$ at the bed node. The value of $\tau$ for 2-D problems can be estimated as foliows:

$\tau_{t}=\mu \gamma_{t} \quad$ with $\quad \gamma_{t}=\frac{1}{2} \frac{\partial v_{t}}{\partial n}=\frac{v_{t}^{k}}{2 h_{k}}$

where $v_{t}^{k}$ is the modulus of the tangential velocity at the node $k$ and $h_{k}$ is a prescribed distance along the normal of the bed node $k$. Typically $h_{k}$ is of the order of magnitude of the smallest fluid element adjacent to node $k$ (Fig. 3).

2. Compute the frictional work originated by the tangential stresses at the bed surface as

$$
W_{f}=\int_{0}^{t} \tau_{t} \gamma_{t} d t=\int_{0}^{t} \frac{\mu}{4}\left(\frac{v_{t}^{k}}{h_{k}}\right)^{2} d t
$$

Eq. (12) is integrated in time as

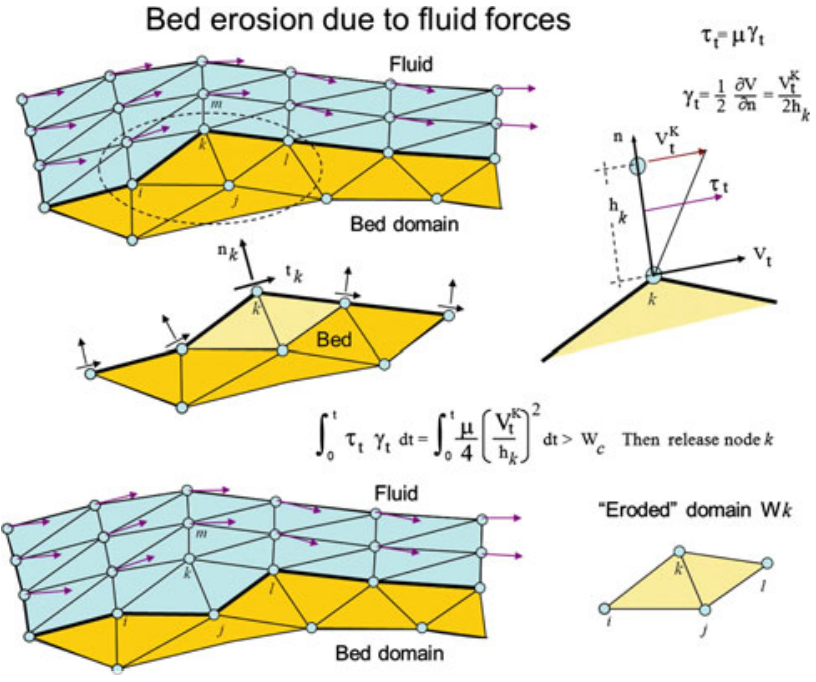

Fig. 3 Modeling of bed erosion with the PFEM by dragging of bed material

$$
{ }^{n} W_{f}={ }^{n-1} W_{f}+\tau_{t} \gamma_{t} \Delta t
$$

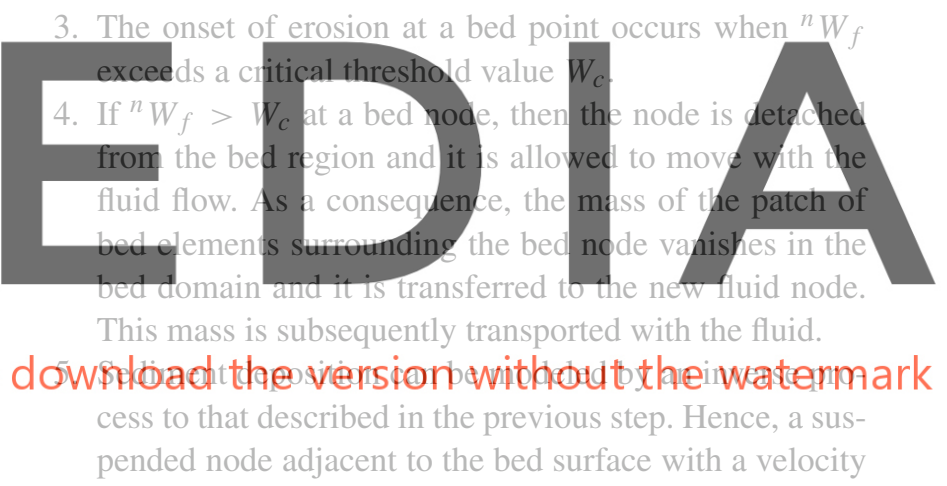

below a threshold value is attached to the bed surface.

Figure 3 shows an schematic view of the bed erosion algorithm described.

\section{Examples}

\subsection{Dragging of rocks by a water stream}

Predicting the critical speed at which a rock will be dragged by a water stream is of great importance in many problems in hydraulic, harbour, civil and environmental engineering.

The PFEM has been successfully applied to the study of the motion of a 1Tn quasi-spherical rock due to a water stream. The rock lays on a collection of rocks that are kept rigid. Frictional conditions between the analyzed rock and the rest of the rocks have been assumed. Figure $4 \mathrm{a}$ shows that a water stream of $1 \mathrm{~m} / \mathrm{s}$ is not able to displace the individual 
(a)

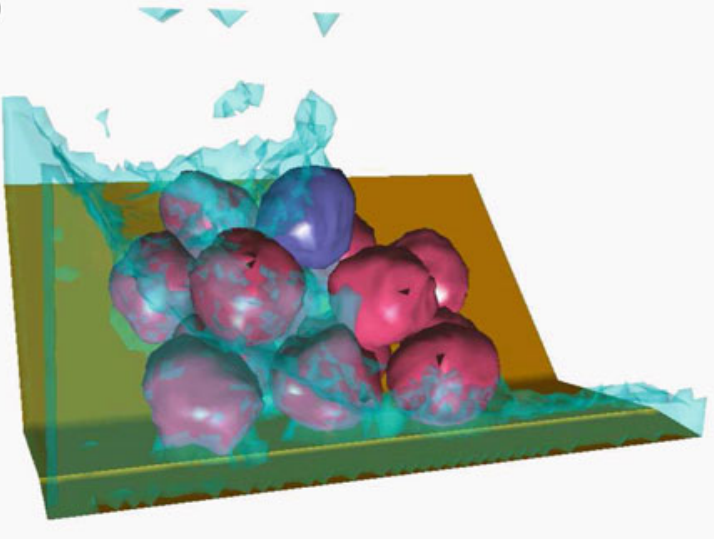

(b)

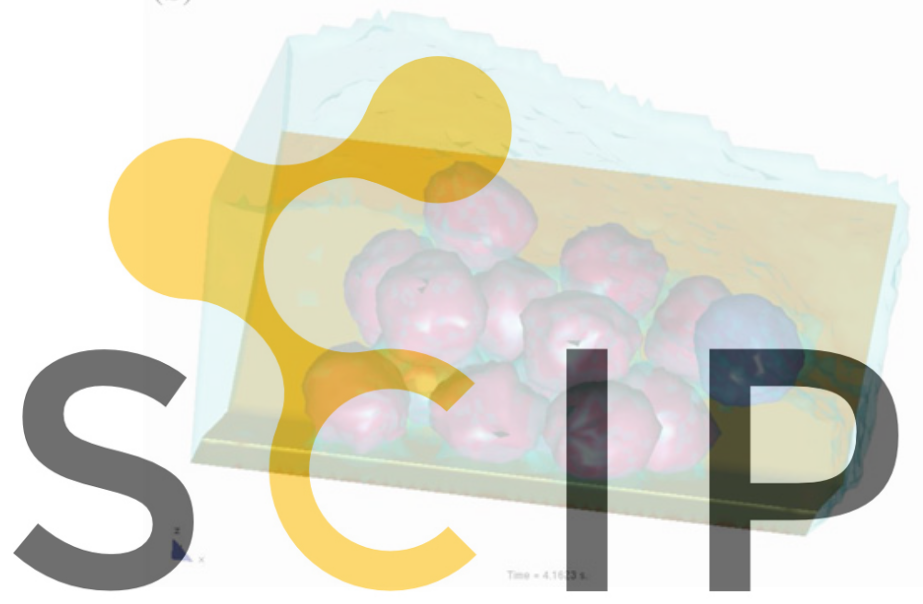

Fig. 4 Study of the drag of an individual rock of 1Tn under a water

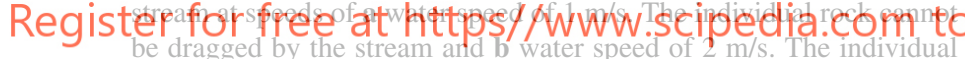
rock is dragged by the stream

rock. An increase of the water speed to $2 \mathrm{~m} / \mathrm{s}$ induces the motion of the rock as shown in Figure $4 \mathrm{~b}$.
8.2 Impact of sea waves on piers and breakwaters

Figures 5 and 6 show the analysis of the effect of breaking waves on two different sites of a breakwater containing reinforced concrete blocks (each one of $4 \times 4 \mathrm{~m}$ ). The figures correspond to the study of Langosteira harbour in A Coruña, Spain using PFEM.

\subsection{Soil erosion problems}

Figure 7 shows the capacity of the PFEM for modelling soil erosion, sediment transport and material deposition in a river bed. The soil particles are first detached from the bed surface under the action of the jet stream. Then they are transported by the flow and eventually fall down due to gravity forces into the bed surface at a downstream point.

Figure 8 shows the progressive erosion of the unprotected part of a breakwater slope in the Langosteira harbour in A Coruña, Spain. The non protected upper shoulder zone is progressively eroded under the sea waves.

Figure 9 displays the progressive erosion and dragging of soil particles in a river bed adjacent to the foot of bridge pile due to the water stream (water is not shown in the figure).

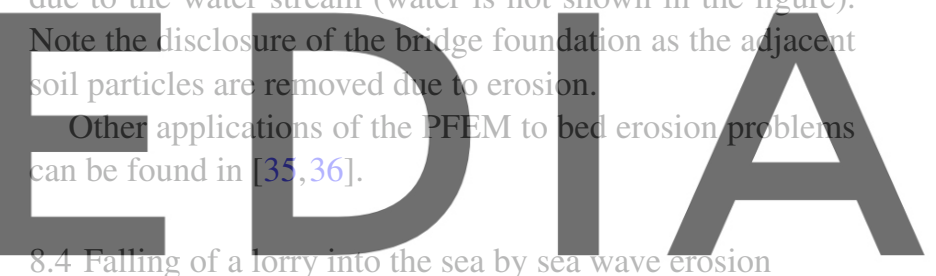

\section{of the road slope}

\section{download the version without the watermark}

Figure 10 shows a representative example of the progressive erosion of a soil mass adjacent to the shore due to sea waves and the subsequent falling into the sea of a 2-D object representing the section of a lorry. The object has been modeled as a rigid solid.
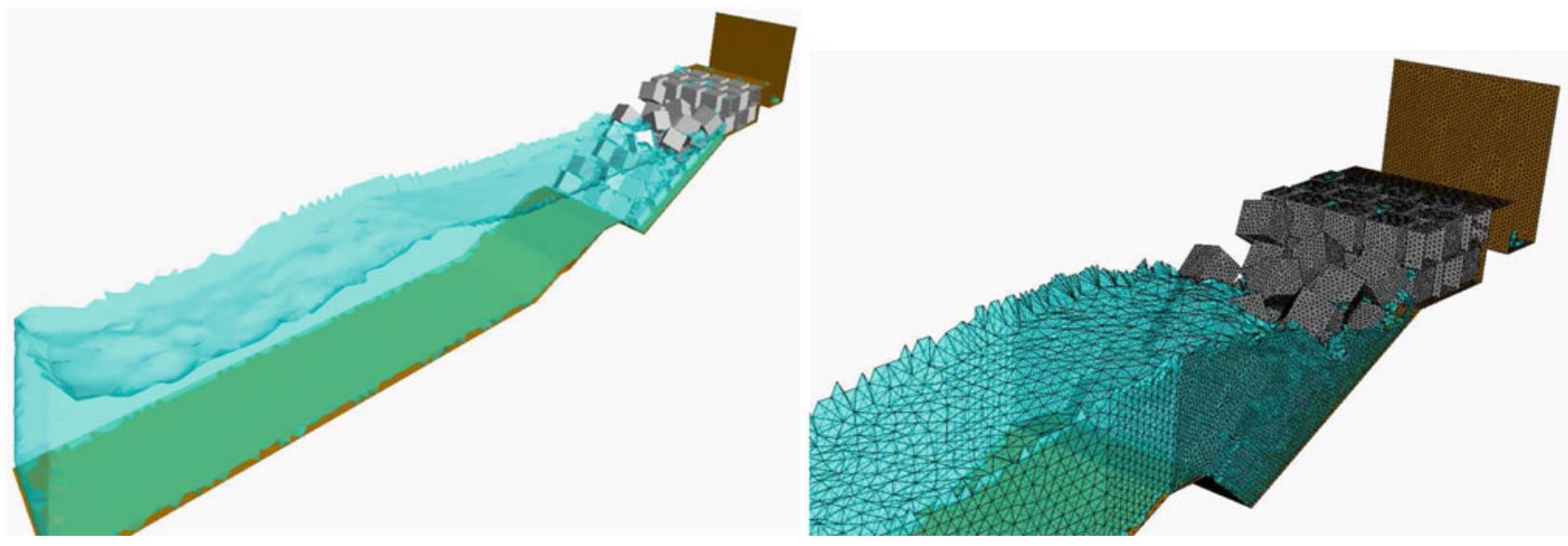

Fig. 5 Breaking waves on breakwater slope containing reinforced concrete blocks. Mesh of 4-noded tetrahedra near the slope 

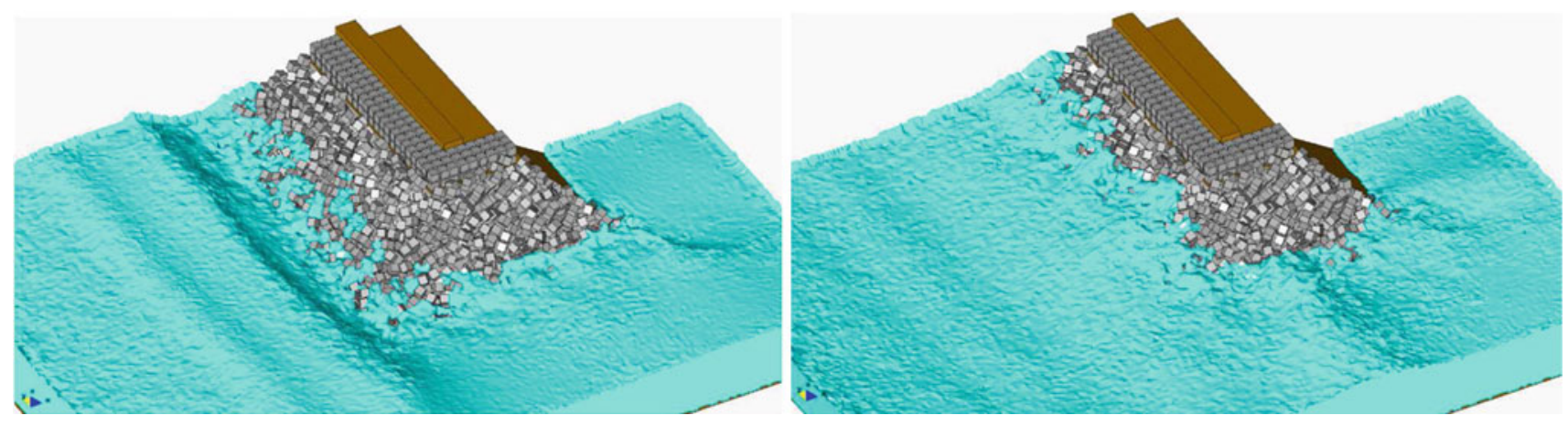

Fig. 6 Study of breaking waves on the edge of a breakwater structure formed by reinforced concrete blocks
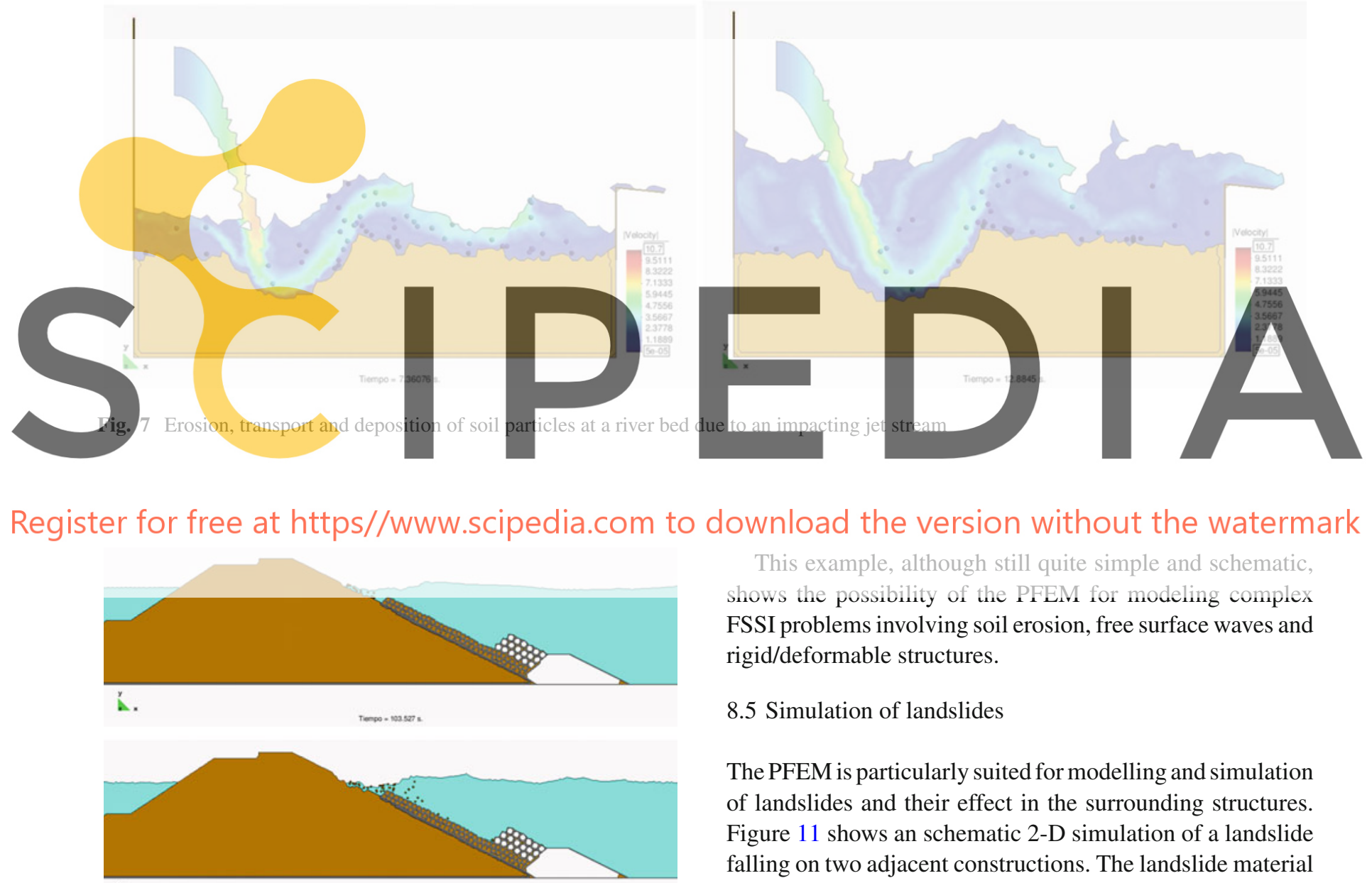

.

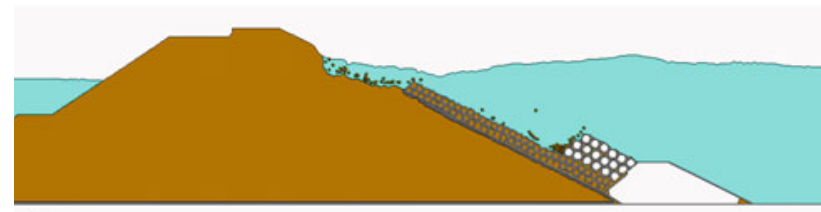

Ĺ.

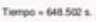

Fig. 8 Erosion of an unprotected shoulder of a breakwater due to sea waves 
Fig. 9 Erosion and dragging of soil particles in a river bed adjacent to the foot of a bridge pile due to a water stream. Water is not shown
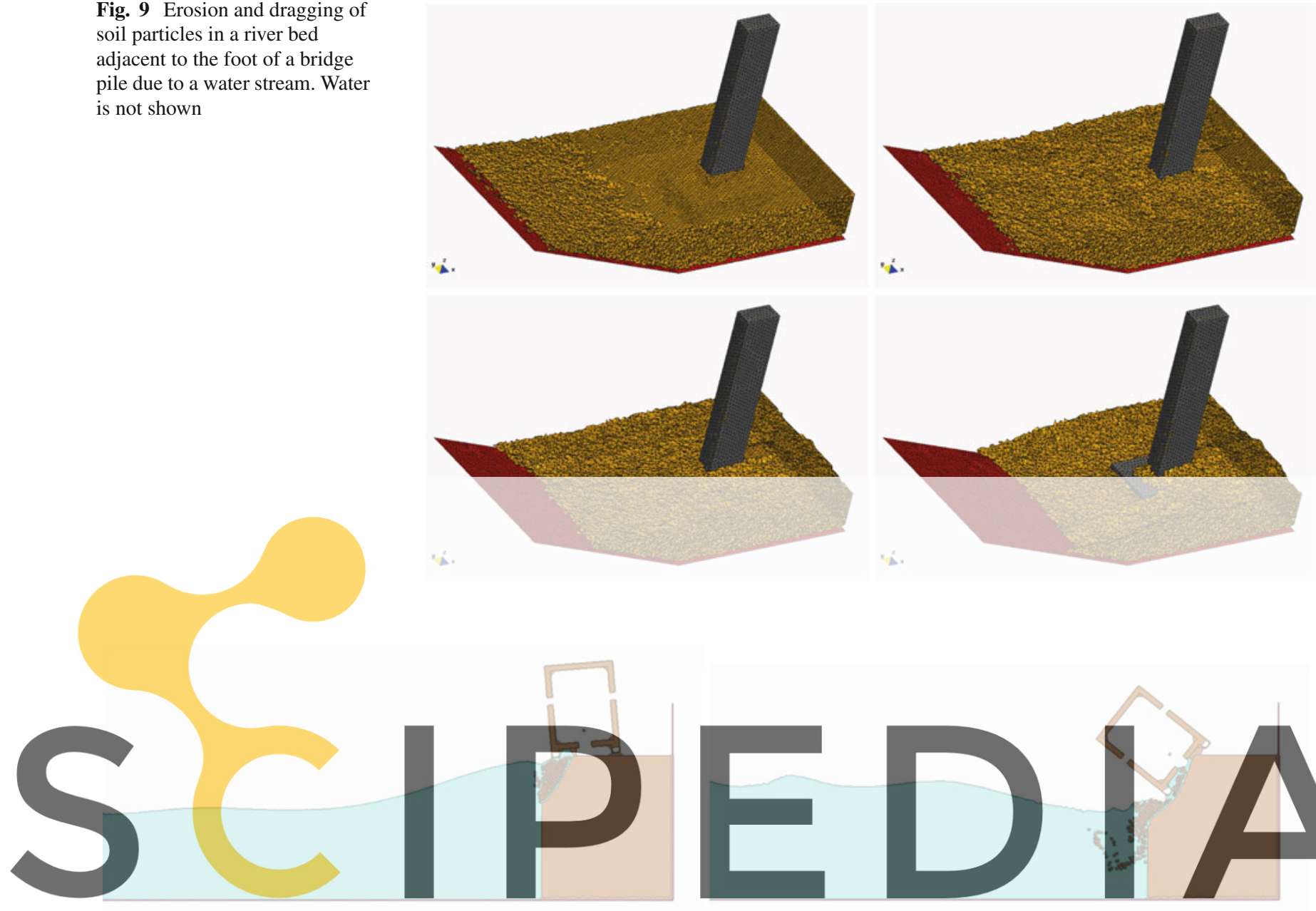

\section{Register for free at https//www.scipedia.com to download the version without the watermark}

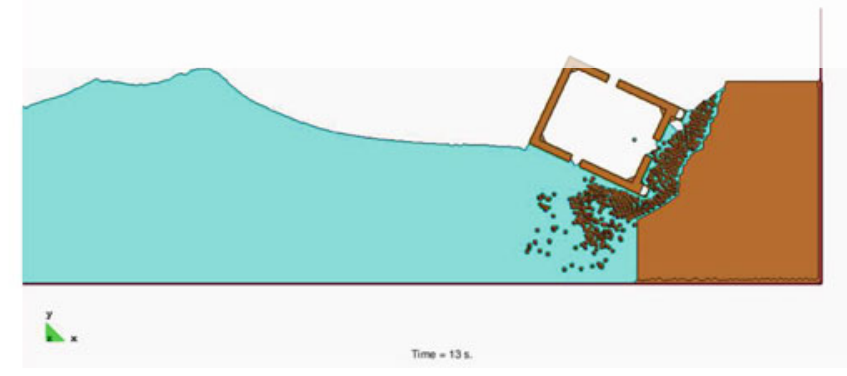

Fig. 10 Erosion of a soil mass due to sea waves and the subsequent falling into the sea of an adjacent lorry

In the example, we present some results of the 3-D analysis of the landslide produced in Lituya Bay (Alaska) on July 9, 1958 (Fig. 12). The landslide was originated by an earthquake and movilized 90 millions tons of rocks that fell on the bay originating a large wave that reached a hight on the opposed slope of $524 \mathrm{~m}$.

Figure 13 show images of the simulation of the landslide with PFEM. The sliding mass has been modelled as a quasi- incompressible continuum with a prescribed shear modulus. No frictional effect between the sliding mass and the underneath soil has been considered. Also the analysis has not taken into account the erosion and dragging of soil material induced by the landslide mass during motion.

PFEM results have been compared with observed values of the maximum water level in the north hill adjacent to the reservoir. The maximum water level in this hill obtained 
Fig. 11 Landslide falling on two constructions 2-D simulation using PFEM
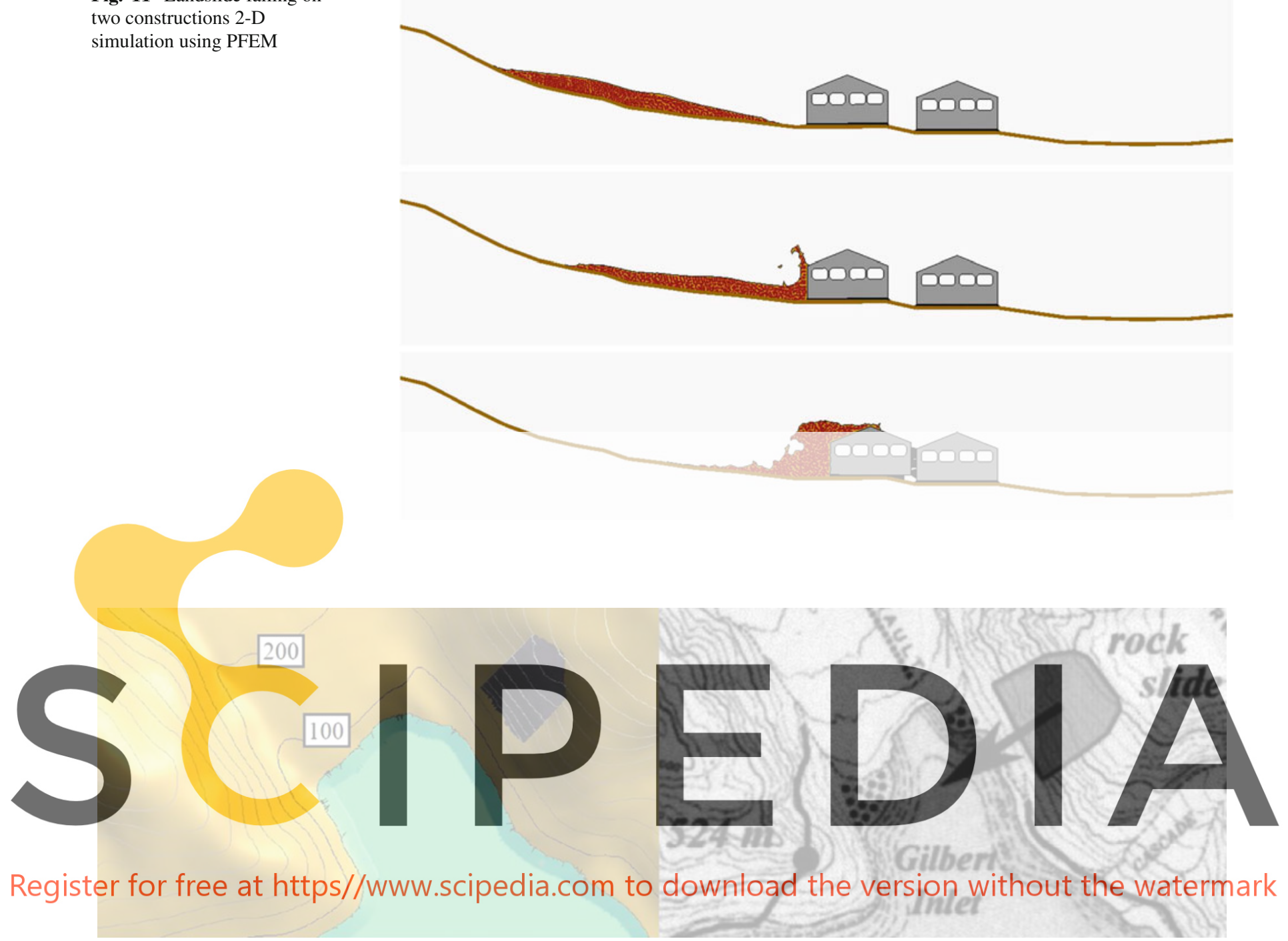

Fig. 12 Lituya Bay landslide. Left geometry for the simulation. Right landslide direction and maximum wave level [13,14]

with PFEM was $551 \mathrm{~m}$. This is $5 \%$ higher than the value of $524 \mathrm{~m}$. observed experimental by $[13,14]$. The maximum height location differs in $300 \mathrm{~m}$ from the observed value [13, 14]. In the south slope the maximum water height observed was $208 \mathrm{~m}$, while the PFEM result (not shown here) was $195 \mathrm{~m}$ (6\% error).

More information on the PFEM solutions of this example can be found in $[38,39]$.

\section{Conclusions}

The particle finite element method (PFEM) is a promising numerical technique for solving fluid-soil-structure interaction (FSSI) problems involving large motion of fluid and solid particles, surface waves, water splashing, frictional contact situations between fluid-solid and solid-solid interfaces and bed erosion, among other complex phenomena. The success of the PFEM lies in the accurate and efficient solution of the equations of an incompressible continuum using an updated Lagrangian formulation and a stabilized finite element method allowing the use of low order elements with equal order interpolation for all the variables. Other essential solution ingredients are the efficient regeneration of the finite element mesh, the identification of the boundary nodes using the Alpha-Shape technique and the simple algorithm to treat frictional contact conditions and erosion/wear at fluid-solid and solid-solid interfaces via mesh generation. The examples presented have shown the potential of the PFEM for solving a wide class of practical FSSI problems in engineering. 


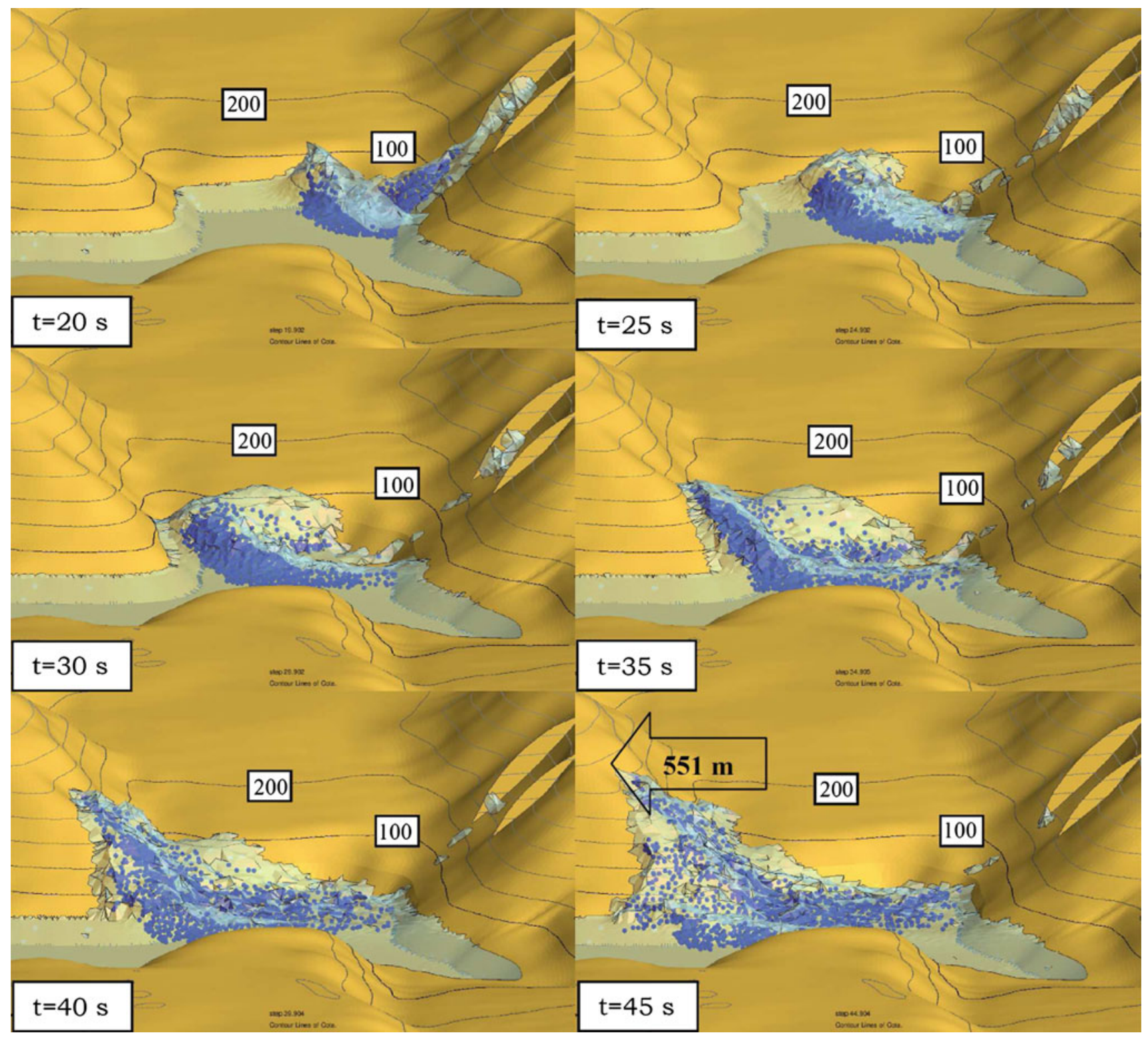

Fig. 13 Lituya Bay landslide. Evolution of the landslide into the reservoir obtained with the PFEM. Maximum level of generated wave (551 $\mathrm{m})$ in the north slope

Acknowledgments This research was partially supported by project SEDUREC of the Consolider Programme of the Ministerio de Educación y Ciencia (MEC) of Spain and the projects SAFECON and REALTIME of the European Research Council of the European Commission (EC). Thanks are also given to the Spanish construction company Dragados for financial support for the study of harbour engineering problems with the PFEM.

\section{References}

1. Archard JF (1953) Contact and rubbing of flat surfaces. J Appl Phys 24(8):981-988

2. Aubry R, Idelsohn SR, Oñate E (2005) Particle finite element method in fluid mechanics including thermal convection-diffusion. Comput Struct 83(17-18):1459-1475
3. Badia S, Codina R (2009) On a multiscale approach to the transient Stokes problem transient subscales and anisotropic spacetime discretizations. Appl Math Comput 207:415-423

4. Baiges J, Codina R (2010) The fixed-mesh ALE approach applied to solid mechanics and fluid-structure interaction problems. Int J Numer Methods Eng 81:1529-1557

5. Bazilevs Y, Calo VM, Cottrell JA, Hughes TJR, Reali A, Scovazzi G (2007) Variational multiscale residual-based turbulence modeling for large eddy simulation of incompressible flows. Comp Methods Appl Mech Eng 197:173-201

6. Bazilevs Y, Hsu M-C, Zhang Y, Wang W, Liang X, Kvamsdal T, Brekken R, Isaksen J (2010) A fully coupled fluid-structure interaction simulation of cerebral aneurysms. Comput Mechan 46: $3-16$

7. Carbonell JM, Oñate E, Suárez B (2010) Modeling of ground excavation with the particle finite element method. J Eng Mechan (ASCE) 136(4):455-463 
8. Codina R (2002) Stabilized finite element approximation of transient incompressible flows using subscales. Comput Methods Appl Mech Eng 191:4295-4321

9. Codina R, Coppola-Owen H, Nithiarasu P, Liu CB (2006) Numerical comparison of CBS and SGS as stabilization techniques for the incompressible Navier-Stokes equations. Int J Numer Methods Eng 66:1672-1689

10. Del Pin F, Idelsohn SR, Oñate E, Aubry R (2007) The ALE/Lagrangian particle finite element method: a new approach to computation of free-surface flows and fluid-object interactions. Comput Fluids 36:27-38

11. Donea J, Huerta A (2003) Finite element method for flow problems. Wiley, Chichester

12. Edelsbrunner H, Mucke EP (1999) Three dimensional alpha shapes. ACM Trans Graphics 13:43-72

13. Fritz HM, Hager WH, Minor HE (2001) Lituya Bay case: rockslide impact and wave run-up. Sci Tsunami Hazards 19(1):3-22

14. Fritz HM, Hager WH, Minor HE (2004) Near field characteristics of landslide generated impulse waves. J Waterway Port Coast Ocean Eng ASCE 130(6):287-302

15. García J, Oñate E (2003) An unstructured finite element solver for ship hydrodynamic problems. J Appl Mech 70:18-26

16. Idelsohn SR, Oñate E, Del Pin F, Calvo N (2002) Lagrangian formulation: the only way to solve some free-surface fluid mechanics problems. In: Mang HA, Rammerstorfer FG, Eberhardsteiner J (eds) 5th World Congress on Comput Mechanics, July 7-12, Vienna, Austria

17. Idelsohn SR, Oñate E, Calvo N, Del Pin F (2003) The meshless finite element method. Int J Numer Methods Eng 58(6):893-912

18. Idelsohn SR, Oñate E, Del Pin F (2003) A lagrangian meshless finite element method applied to fluid-structure interaction problems. Comput Struct 81:655-671

19. Idelsohn SR, Calvo N, Oñate E (2003) Polyhedrization of an arbitrary point set. Comput Methods Appl Mech Eng 192(2224):2649-2668

20. Idelsohn SR, Oñate E, Del Pin F (2004) The particle finite element method: a powerful tool to solve incompressible flows with free-surfaces and breaking waves. Int J Numer Methods Eng 61: 964-989

21. Idelsohn SR, Oñate E, Del Pin F, Calvo N (2006) Fluid-structure interaction using the particle finite element method. Comput Methods Appl Mech Eng 195:2100-2113

22. Idelsohn SR, Marti J, Limache A, Oñate E (2008) Unified Lagrangian formulation for elastic solids and incompressible fluids: application to fluid-structure interaction problems via the PFEM. Comput Methods Appl Mech Eng 197:1762-1776

23. Idelsohn SR, Mier-Torrecilla M, Oñate E (2009) Multi-fluid flows with the particle finite element method. Comput Methods Appl Mech Eng 198:2750-2767

24. Johnson AA, Tezduyar TE (1999) Advanced mesh generation and update methods for 3-D flow simulations. Comput Mech 23:130 143

25. Kovacs A, Parker G (1994) A new vectorial bedload formulation and its application to the time evolution of straight river channels. J Fluid Mech 267:153-183

26. Larese A, Rossi R, Oñate E, Idelsohn SR (2008) Validation of the particle finite element method (PFEM) for simulation of free surface flows. Eng Comput 25(4):385-425

27. Löhner R (2008) Applied CFD techniques. Wiley, Chichester

28. Löhner R, Yang C, Oñate E (2007) Simulation of flows with violent free surface motion and moving objects using unstructured grids. Int J Numer Methods Fluids 153:1315-1338
29. Oñate E (1998) Derivation of stabilized equations for advectivediffusive transport and fluid flow problems. Comput Methods Appl Mech Eng 151:233-267

30. Oñate $E$ (2004) Possibilities of finite calculus in computational mechanics. Int J Numer Methods Eng 60(1):255-281

31. Oñate E, García J (2001) A finite element method for fluidstructure interaction with surface waves using a finite calculus formulation. Comput Methods Appl Mech Eng 191:635-660

32. Oñate E, Idelsohn SR, Del Pin F, Aubry R (2004) The particle finite element method: an overview. Int J Comput Methods 1(2):267-307

33. Oñate E, Valls A, García J (2006) FIC/FEM formulation with matrix stabilizing terms for incompressible flows at low and high Reynold's numbers. Comput Mechan 38(4-5):440-455

34. Oñate E, García J, Idelsohn SR, Del Pin F (2006) FIC formulations for finite element analysis of incompressible flows Eulerian, ALE and Lagrangian approaches.. Comput Methods Appl Mech Eng 195(23-24):3001-3037

35. Oñate E, Celigueta MA, Idelsohn SR (2006) Modeling bed erosion in free surface flows by the particle finite element method. Acta Geotechnia 1(4):237-252

36. Oñate E, Idelsohn SR, Celigueta MA, Rossi R (2008) Advances in the particle finite element method for the analysis of fluidmultibody interaction and bed erosion in free surface flows. Comput Methods Appl Mech Eng 197(19-20):1777-1800

37. Oñate E, Rossi R, Idelsohn SR, Butler K (2010) Melting and spread of polymers in fire with the particle finite element method. Int J Numer Methods Eng 81(8):1046-1072

38. Oñate E, Salazar F, Morán R (2011) Modeling of landslides into reservoir with the particle finite element method. Research Report CIMNE No. PI355. Submitted to Int J Numer Methods Geomechan

39. Salazar F, Oñate E, Morán R (2011) Modelación numérica de deslizamientos de ladera en embalses mediante el método de partículas y elementos finitos (PFEM). Rev Int Mét Num Cálc Dis Ing. Accepted for publication

40. Takizawa K, Tezduyar TE (2011) Multiscale space-time fluidstructure interaction techniques. Comput Mechan. doi:10.1007/ s00466-011-0571-z

41. Tezduyar TE, Mittal S, Ray SE, Shih R (1992) Incompressible flow computations with stabilized bilinear and linear equal-orderinterpolation velocity-pressure elements. Comput Methods Appl Mech Eng 95:221-242

42. Tezduyar TE (2007) Finite elements in fluids: special methods and enhanced solution techniques. Comput Fluids 36:207-223

43. Wan CF, Fell R (2004) Investigation of erosion of soils in embankment dams. J Geotechn Geoenviron Eng 130:373-380

44. Yabe T, Takizawa K, Tezduyar TE, Im H-N (2007) Computation of fluid-solid and fluid-fluid interfaces with the CIP method based on adaptive Soroban Grids: an overview. Int J Numer Methods Fluids 54:841-853

45. Zienkiewicz OC, Jain PC, Oñate E (1978) Flow of solids during forming and extrusion: some aspects of numerical solutions. Int J Solids Struct 14:15-38

46. Zienkiewicz OC, Taylor RL, Zhu JZ (2005) The finite element method. Its basis and fundamentals. Elsevier, Oxford

47. Zienkiewicz OC, Taylor RL (2005) The finite element method for solid and structural mechanics. Elsevier, Oxford

48. Zienkiewicz OC, Taylor RL, Nithiarasu P(2006) The finite element method for fluid dynamics. Elsevier, Oxford 